\title{
Pup Suckling Is More Rewarding Than Cocaine: Evidence from Functional Magnetic Resonance Imaging and Three-Dimensional Computational Analysis
}

\author{
Craig F. Ferris, ${ }^{1}$ Praveen Kulkarni, ${ }^{2}$ John M. Sullivan Jr, ${ }^{2}$ Josie A. Harder, ${ }^{3}$ Tara L. Messenger, ${ }^{1}$ and Marcelo Febo ${ }^{1}$ \\ ${ }^{1}$ Center for Comparative Neuroimaging, University of Massachusetts Medical School, Worcester, Massachusetts 01655, ${ }^{2}$ Department of Mechanical \\ Engineering, Worcester Polytechnic Institute, Worcester, Massachusetts 01609, and ${ }^{3}$ School of Pharmacy, University of Bradford, West Yorkshire BD7 1DP, \\ United Kingdom
}

\begin{abstract}
Nursing has reciprocal benefits for both mother and infant, helping to promote maternal behavior and bonding. To test the "rewarding" nature of nursing, functional magnetic resonance imaging was used to map brain activity in lactating dams exposed to their suckling pups versus cocaine. Suckling stimulation in lactating dams and cocaine exposure in virgin females activated the dopamine reward system. In contrast, lactating dams exposed to cocaine instead of pups showed a suppression of brain activity in the reward system. These data support the notion that pup stimulation is more reinforcing than cocaine, underscoring the importance of pup seeking over other rewarding stimuli during lactation.
\end{abstract}

Key words: accumbens; cocaine; magnetic; maternal; motivation; nigrostriatal; prefrontal; substantia nigra; suckling

\section{Introduction}

The mother-infant bond is a critical event, fostering maternal behavior and enhancing infant survival by providing nutrients and warmth. The initiation and maintenance of maternal behavior in rodents is a complex interaction between the endocrine status of the dam before and after parturition and the continued interaction with and stimulation from pups until weaning $(\mathrm{Nu}-$ man, 1994). Hormones combine with the physiology of parturition to foster maternal behavior in first-time mothers. However, from postpartum day 4 through postpartum day 20, the maintenance of maternal behavior is more strongly influenced by learning and the tactile and odor stimuli coming from the pups.

Pups have reinforcing properties that are so salient, they can compete with self-administration of cocaine (Hecht et al., 1999). Over several studies, Fleming and coworkers characterized the reinforcing nature of pups in maternal behavior (Fleming et al., 1994; Magnusson and Fleming, 1995; Lee et al., 2000), ultimately showing that postpartum dams will bar press for pups in an operant response paradigm (Lee et al., 2000). The reinforcing property of pups is highest between postpartum day 1 and postpartum day 7 but declines dramatically by postpartum day 10, evidence

Received Aug. 2, 2004; revised 0ct. 4, 2004; accepted 0ct. 29, 2004.

This work was funded by a grant from the National Institute of Mental Health (NIMH), Behavioral and Integrative Neuroscience Grant R01 MH 52280, and the National Institute on Drug Abuse (NIDA), Division of Basic Research, Behavioral Neurobiology Grant R01 DA13517. Its contents are solely the responsibility of the authors and do not necessarily represent the official views of NIDA. We thank Dr. Thomas Insel from NIMH for his encouragement and advice on the initiation and design of these studies.

Correspondence should be addressed to Dr. Craig F. Ferris, Department of Psychiatry, Center for Comparative Neuroimaging, University of Massachusetts Medical School, 55 Lake Avenue North, Worcester, MA 01655. E-mail: craig.ferris@umassmed.edu.

DOI:10.1523/JNEUROSCI.3156-04.2005

Copyright $\odot 2005$ Society for Neuroscience $\quad$ 0270-6474/05/250149-08\$15.00/0 that perhaps the dams' appetitive motivation changes over time. This notion was examined more closely by Mattson et al. (2001, 2003) using a place-preference paradigm to investigate the reinforcing properties of cocaine versus pups in lactating dams at different postpartum periods. On postpartum day 8 , dams prefer the pup cue-associated location compared with postpartum days 10 and 16, in which they prefer the cocaine cue-associated chamber.

Activation of the dopaminergic mesocorticolimbic system or reward pathway is hypothesized to be part of a central mechanism contributing to the reinforcing property of pups. The origin of the dopaminergic innervations to the major components of the reward system, nucleus accumbens and prefrontal cortex, is the ventral tegmental area (VTA). Lesioning the VTA reduces maternal behavior (Pedersen et al., 1994). Depleting dopamine levels in this VTA-accumbens projection with a neurotoxin reduces pup retrieval (Hansen et al., 1991a,b), and direct electrolytic lesion of the accumbens alters maternal memory over the postpartum period (Lee et al., 1999). The reinforcing properties of pups during the postpartum period can be blocked with a dopamine receptor antagonist (Fleming et al., 1994). The present studies were undertaken to test the hypothesis that the reinforcing nature of pup stimulation involves activation of the reward pathway and that during early lactation, the reward pathway is more sensitive to pups than cocaine.

\section{Materials and Methods}

Animals. Virgin Sprague Dawley female rats, 80-90 d of age, were obtained from Charles River Laboratories (Wilmington, MA) and were housed individually in $48 \times 25 \times 20 \mathrm{~cm}$ Plexiglas cages. Animals were maintained on a $12 \mathrm{hr}$ light/dark cycle with lights on at 7:00 A.M. and were provided with food and water ad libitum. Before breeding, animals were acclimated to the rodent 
restrainer and the imaging protocol, as described below. All dams studied had a minimum litter of 10 pups. The use of animals adhered to the Animal Welfare Act, Principles for Use of Animals, and Public Health Service Policy on Humane Care and Use of Laboratory Welfare.

Animal restrainer. Studies were performed with a multiconcentric, dual-coil small animal restrainer (Insight Neuroimaging Systems, Worcester, MA). In brief, just before the imaging session, dams were anesthetized with $4 \%$ isoflurane. A topical anesthetic of 5\% lidocaine gel was applied to the skin and soft tissue around the ear canals and over the bridge of the nose. A plastic semicircular headpiece with blunted ear supports that fit into the ear canals was positioned over the ears. The head was placed into a cylindrical head holder with the animal's canines secured over a bite bar and the ears positioned inside the head holder with adjustable screws fitted into lateral sleeves. An adjustable surface coil built into the head holder was pressed firmly on the head and locked into place. The body of the animal was placed into a body restrainer. The body restrainer "floats" down the center of the chassis connecting at the front and rear endplates and is buffered by rubber gaskets. The head piece locks into a mounting post on the front of the chassis. This design isolates all of the body movements from the head restrainer and minimizes motion artifact. Once the animal was positioned in the body holder, a volume coil was slid over the head restrainer and locked into position.

Acclimating animals to the imaging protocol. Before imaging, animals were routinely acclimated to the restrainer and the imaging. Animals were anesthetized with isoflurane as described above for securing the animal into the restrainer. When fully conscious, the restraining unit was placed into a black opaque tube "mock scanner" with a tape recording of a magnetic resonance imaging (MRI) pulse sequence for $90 \mathrm{~min}$ to simulate the bore of the magnet and an imaging protocol. This procedure was repeated every other day for $4 \mathrm{~d}$ and has been used successfully in several awake imaging studies to acclimate rats to the restrainer and imaging protocol (Brevard et al., 2003; Tenney et al., 2003; Febo et al., 2004). Rats show a significant decline in body temperature, motor movements, heart rate, and plasma corticosterone levels when comparing the first to the last acclimation periods.

Imaging protocol. Experiments were conducted in a Bruker (Billerica, MA) BioSpec 4.7 $\mathrm{T}, 40 \mathrm{~cm}$ horizontal magnet (Oxford Instruments, Oxford, UK) equipped with a Bruker BioSpec console and a 20 Gauss/cm magnetic field gradient insert (inner diameter, $12 \mathrm{~cm}$ ) capable of a $120 \mu \mathrm{sec}$ rise time (Bruker). Radiofrequency signals were sent and received with the dual-coil electronics built into the animal restrainer (Ludwig et al., 2003). The volume coil for sending radio frequency signal features an eightelement microstrip line configuration that, in conjunction with an outer copper shield, forms a transverse electromagnetic resonator structure. The arch-shaped geometry of the receiving surface coil provides excellent coverage and high signal-to-noise ratio. To prevent mutual coil interference, the volume and surface coils were actively tuned and detuned.

Functional images were acquired using a multislice fast spin echo se-

\section{Pup Stimulation in Lactating Dams}
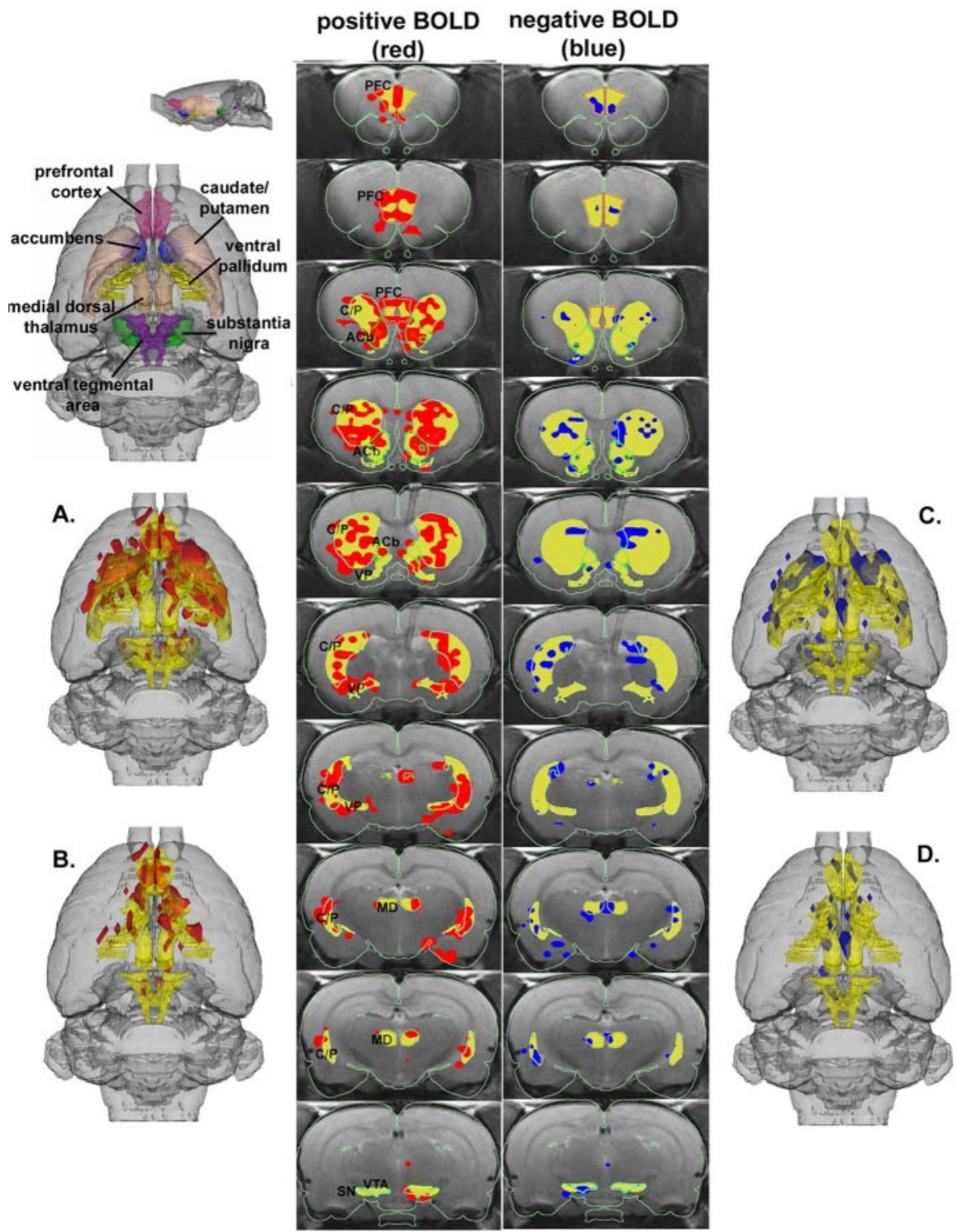

Figure 1. 3D activational map showing pup stimulation in lactating dams. Top left picture is a translucent shell of the brain viewed from a dorsal perspective showing color-coded VOls corresponding to anatomical geometries of the mesocorticolimbic and nigrostriatal dopamine systems. For orientation, a smaller rendering of the same picture is shown from a lateral perspective. Colors have been melded into a single functional VOI (yellow) showing localization of positive (red) and negative (blue) BOLD signal changes with pup stimulation. $A$ includes both dopamine systems, whereas $B$ has masked the caudate-putamen (C/P) and substantia nigra (SN) comprising the nigrostriatal dopamine system, revealing the mesocorticolimbic or reward system. C and $D$ are the corresponding negative BOLD images for each functional volume, respectively. The middle columns show traditional activation maps of contiguous brain sections with labeled regions of interest. The significantly activated voxels are the composite of all six subjects overlaid on one subject used as an anatomical template. The location of every voxel in any subject whose $p$ value was $<0.05$ after false-positive filtering for $\pm B O L D$ signal change is included in the composite analysis and located within the region of interest. PFC, Prefrontal cortex; $A C b$, accumbens; VP, ventral pallidum; MD, medial dorsal thalamus.

quence. A single data acquisition acquired 12, $1.2 \mathrm{~mm}$ slices in $6 \mathrm{sec}$ [field of view (FOV), $3.0 \mathrm{~cm}$; data matrix, $64 \times 64$; repetition time (TR), $1.4 \mathrm{sec}$; echo time (TE), 7 msec; number of excitations (NEX), 1]. This sequence was repeated 80 times in an $8 \mathrm{~min}$ imaging session consisting of $3 \mathrm{~min}$ of baseline data followed by 5 min of stimulation data. At the end of each imaging session, a high-resolution anatomical data set was collected using the rapid acquisition with relaxation enhancement pulse sequence (12 slices; $1.2 \mathrm{~mm}$; FOV, $3.0 \mathrm{~cm} ; 256 \times 256$; TR, $2.1 \mathrm{sec}$; TE, $12.4 \mathrm{msec}$; NEX, 12; acquisition time, $13 \mathrm{~min}$ ).

Experimental design. Three stimulation conditions were studied using six animals for each stimulus. In one condition, lactating dams between 


\section{Cocaine Stimulation in Virgin Female}

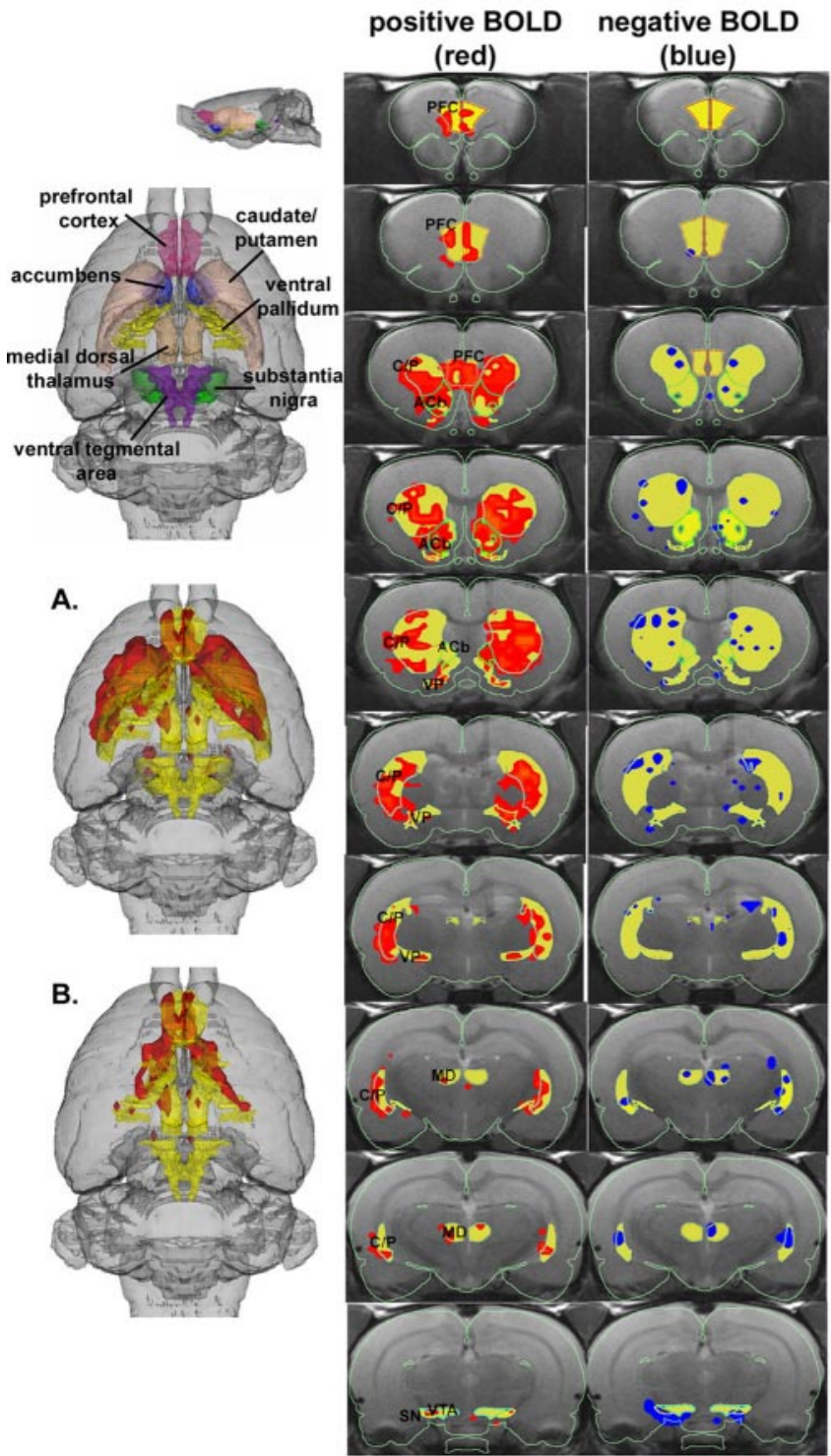

pulled. When the shield was pulled away, the pups were exposed to the six hindlimb nipples and would begin suckling. We were able to visually confirm when pups came onto the most caudal teats but were not able to determine whether all teats were suckled during the $5 \mathrm{~min}$ stimulation period. In all six pup stimulation studies, suckling occurred within seconds of removing the shield. To promote suckling in the magnet, dams were prevented from having physical contact with their pups for $2 \mathrm{hr}$ before imaging. This was accomplished by inverting a shallow perforated Plexiglas box over the huddled pups. It should also be noted that Mattson et al. (2001) showed that a 2-3 hr separation was necessary for a female to learn conditioned place preference for pups.

The technology and methods for imaging cocaine stimulation of the mesocorticolimbic dopamine system in fully conscious rats have been published previously (Febo et al., 2004). In brief, within 2-5 $\mathrm{min}$ of ICV cocaine at a threshold dose of $20 \mu \mathrm{g}$, there is a significant increase in stereotypic behavior and release of dopamine from the accumbens. This route of administration is advantageous because the brain is exposed to a known amount of cocaine, and the action of the drug is limited to the CNS. The complications associated with interpreting changes in blood-oxygenation-level-dependent (BOLD) signal secondary to the effects of cocaine on cardiovascular and respiratory function are avoided. While anesthetized with $4 \%$ isoflurane, the skin over the skull is excised, exposing the landmark suture bregma. A 26 gauge cannula of polyethylene tubing (PE-10; outer diameter) is placed into the lateral cerebroventricle $2.5 \mathrm{~mm}$ lateral to bregma and $5 \mathrm{~mm}$ below dura and secured to the skull with surgical glue. The area of the incision is swabbed with 5\% lidocaine gel. The length of the cannula tubing is $\sim 30 \mathrm{~cm}$, allowing for cocaine injection via a $20 \mu \mathrm{l}$ glass Hamilton syringe connected at the end of the tubing. The flexible cannula makes it possible to press the surface coil onto the head when the animal is positioned in the head holder. The volume of $20 \mu \mathrm{l}$ allows for labeled contrast agent to reach all areas of the reward system within 30 sec after a bolus ICV injection.

Data analysis. Anatomy images for each sub-

postnatal day 4 (P4) and P8 were exposed to their pups for a 5 min stimulation period. In the second condition, virgin females were exposed to a bolus intraventricular (ICV) injection of cocaine $(20 \mu \mathrm{g} / 20 \mu \mathrm{l})$, and in the third condition, lactating dams between $\mathrm{P} 4$ and $\mathrm{P} 8$ were exposed to ICV-injected cocaine. Data acquisition for control ( $3 \mathrm{~min}$ ) and stimulation ( $5 \mathrm{~min}$ ) periods was continuous. Over the course of the studies, it was discovered that both pup and cocaine stimulation could be run in the same lactating dam during a single imaging session. Consequently, two of the six lactating dams provided data for each stimulation condition. In these two cases, the presentation of the different stimuli was counterbalanced and separated by $45 \mathrm{~min}$. All animals were temporally anesthetized with $4 \%$ isoflurane, secured in the holder, and allowed to awaken as described previously. For pup stimulation studies, the hindlimbs of the dams were loosely tethered and raised just above the floor of the body tube. This provided a visual inspection of the ventrum from outside the magnet and prevented the dam from kicking and injuring the pups during suckling. A cradle containing six pups was positioned under the dam in the magnet. The body tube had a window exposing the dam's ventrum to the pups. A thin plastic shield separated the pups from the mother. The shield was carefully positioned so it did not touch the mother when ject were obtained at a resolution of $256^{2} \times 12$ slices and a FOV of $30 \mathrm{~mm}$ with a slice thickness of $1.2 \mathrm{~mm}$. Subsequent functional imaging was performed at a resolution of $64^{2} \times 12$ slices with the same FOV and slice thickness. Eighty data acquisitions were performed with a period of $6 \mathrm{sec}$ each for a total lapse time of $480 \mathrm{sec}$ or $8 \mathrm{~min}$. The first 30 acquisitions were used for acclimation and control. The monitoring continued for an additional 50 acquisitions, with the stimulus (cocaine or suckling pups) being introduced at time period $3 \mathrm{~min}$. After the functional sequences, repeated anatomy images were collected and compared with the initial anatomy image sets. Motion artifact was assessed by: (1) subtraction of anatomical data across the imaging session, (2) qualitative analysis of time-series movies looking for voxel displacement, and (3) analysis of raw data time series for course spikes. The time-series movies correlated with course spike activity. The multiple data sets collected from these imaging sessions showed very little motion artifact using these criteria. There were no discernable differences in motion artifact as a function of stimulus type. On rare occasions, there would be a course spike, usually caused by movement of the mouth such as in swallowing. The data for these images were excluded. 
Each subject was registered or aligned to a fully segmented rat brain atlas that delineates $>1200$ distinct anatomical subvolumes within the brain based on two-dimensional atlas textbooks (Paxinos and Watson 1991; Swanson 1998). These detailed regions are collected into 12 major regions of the brain (e.g., amygdaloid complex, cerebrum, cerebellum, hypothalamus, etc.). A functional atlas of the mesocorticolimbic dopamine system or reward pathway was developed from numerous anatomical regions. The alignment process began by outlining the brain perimeters for each slice of the anatomy image sets. A marching cube algorithm with automated linearization creates accurate three-dimensional (3D) surface shells for each subject (Wu and Sullivan 2003; Huang et al., 2004). This enhanced surface generation strategy eliminates the characteristic stair-stepped behavior of the marching cube algorithms while simultaneously increasing the accuracy of the geometry representation. These anatomy shells were aligned to the atlas shell. The affine registration involved translation, rotation, and scaling in all three dimensions, independently.

The matrices that transformed the subject's anatomy shells to the atlas space were used to embed each slice within the atlas. All transformed pixel locations of the anatomy images were tagged with the segmented atlas major and minor regions creating a fully segmented representation of each subject. The inverse transformation matrix $\left[T_{i}\right]^{-1}$ for each subject (i) was also calculated. An interactive graphic user interface facilitated these shell alignments (Zhang et al., 2003). Approximately 20 min per subject were required to create the slice perimeters, run the marching cube, align the geometries, and create the final segmented anatomy.

Statistical $t$ tests were performed on each subject within their original coordinate system. The control window was the first 30 time periods. The stimulation window was the remaining 50 time periods. The $t$ test statistics used a 95\% confidence level, two-tailed distributions, and heteroscedastic variance assumptions. As a result of the multiple $t$ test analyses performed, a false-positive detection controlling mechanism was introduced (Genovese et al., 2002). This subsequent filter guaranteed that, on average, the false-positive detection rate was below our cutoff of 0.05 . The formulation of the filter satisfied the following expression:

$$
P_{(i)} \leq \frac{i}{V} \frac{q}{c(V)}
$$

where $P(i)$ is the $p$ value based on the $t$ test analysis. Each pixel $(i)$ within the region of interest (ROI) containing $(V)$ pixels was ranked based on its probability value. The false-positive filter value $q$ was set to be 0.05 for our analyses, and the predetermined constant $c(V)$ was set to unity, which is appropriate for data containing Gaussian noise such as functional MRI (fMRI) data (Genovese et al., 2002). These analysis settings provided conservative estimates for significance. Those pixels deemed statistically significant retained their percentage change values (stimulation mean minus control mean) relative to control mean. All other pixel values were set to zero.

The segmented atlas was cropped and rendered onto 12 slices of $256^{2}$ resolution corresponding to the FOV of the subjects. This cropped atlas served as the segmented composite with coordinates for row, column, and slice. A statistical composite was created for each group. A Bonferroni correction factor was used to maintain an overall uncertainty level of 0.05 for comparisons among the three groups (Petruccelli et al., 1999). The individual analyses were summed within groups. The composite statistics were built using the inverse transformation matrices. Each composite pixel location (i.e., row, column, and slice), premultiplied by $\left[T_{i}\right]^{-1}$, mapped it within a voxel of subject $(i)$. A trilinear interpolation of the subject's voxel values (percentage change) determined the statistical contribution of subject (i) to the composite (row, column, and slice) location. The use of $\left[T_{i}\right]^{-1}$ ensured that the full volume set of the composite was populated with subject contributions. The average value from all subjects within the group determined the composite value.

The BOLD response maps of the composite were somewhat broader in their spatial coverage than in an individual subject. Consequently, the magnitudes of the statistical values were commensurately reduced. This outcome was a natural consequence of the inability to align each subject within a pixel resolution. However, the subjects were aligned very well at the resolution of the ROIs. The time history graphs for each composite region were based on the weighted average of each subject for that region, as follows:

$$
\text { Activated composite pixels }=\frac{\sum_{i=1}^{i=N} \text { Activated pixels subject }(i)}{N}
$$

Composite percentage change

$$
=\frac{\left.\sum_{i=1}^{i=N} \text { Activated pixels subject }(i) \times \text { Percentage change }(i)\right)}{\text { Activated composite pixels }}
$$

where $N$ is the number of subjects.

\section{Results}

Three-dimensional activational maps for each stimulus condition are shown in Figures 1-3. Each image is a composite of six subjects, fully registered and segmented for volumes of interest (VOI) comprising the mesocorticolimbic and nigrostriatal dopamine systems. Pup stimulation causes areas of positive BOLD activation localized to both dorsal (caudate-putamen) and ventral (accumbens) striatum and prefrontal cortex (Fig. 1, left column). These areas of activation are very similar to that observed with cocaine stimulation in virgin females (Fig. 2) but not cocaine stimulation in lactating dams (Fig. 3). The volume of activation of the primary forebrain areas comprising the reward system (i.e., accumbens and prefrontal cortex) can be viewed in a $3 \mathrm{D}$ model (Fig. 3B) for each stimulus condition. The most obvious difference among the three stimulus conditions in terms of positive BOLD signal is the absence of activation in the prefrontal cortex in lactating dams exposed to cocaine (Figs. $1 \mathrm{~B}, 2 \mathrm{~B}, 3 \mathrm{~B}$ ). Although the activation of the accumbens appears to be comparable between lactating dams stimulated with pups versus cocaine in the $3 \mathrm{D}$ presentation, careful investigation of the contiguous coronal sections (Figs. 1-3, left columns) shows that the cocaine stimulation is limited to the most caudal-dorsal accumbens (putative core), whereas pups stimulation is much broader, including rostral-ventral accumbens (putative shell).

Pups stimulation also caused modest but significant changes in negative BOLD signal, as shown in Figure 1, $C$ and $D$ (right column). These negative BOLD data were very similar to cocaine stimulation in virgin females (Fig. 2). One of the more striking differences among stimulation conditions was the robust negative BOLD signal change observed throughout the mesocorticolimbic and nigrostriatal dopaminergic systems in lactating dams exposed to cocaine (Fig. 3).

A quantitative analysis of the volumes of activation shown in Figures 1-3 is presented in Figure 4. Bar graphs show the median plus minimum and maximum number of voxels in the accumbens, prefrontal cortex, caudate-putamen, medial dorsal thalamus, and ventral tegmental area for each stimulus condition. There were no significant differences in voxel numbers for the substantia nigra or ventral pallidum between any of the stimulus conditions (data not shown). Also, there was no significant difference in the number of positive or negative BOLD voxels between pup stimulation and cocaine stimulation in virgin females. Consequently, all noted significant differences are positive or negative BOLD comparisons between pups-dam and cocainevirgin versus cocaine-dams. The statistics for these group comparisons used the Bonferroni correction to maintain an overall 


\section{Cocaine Stimulation in Lactating Dams}
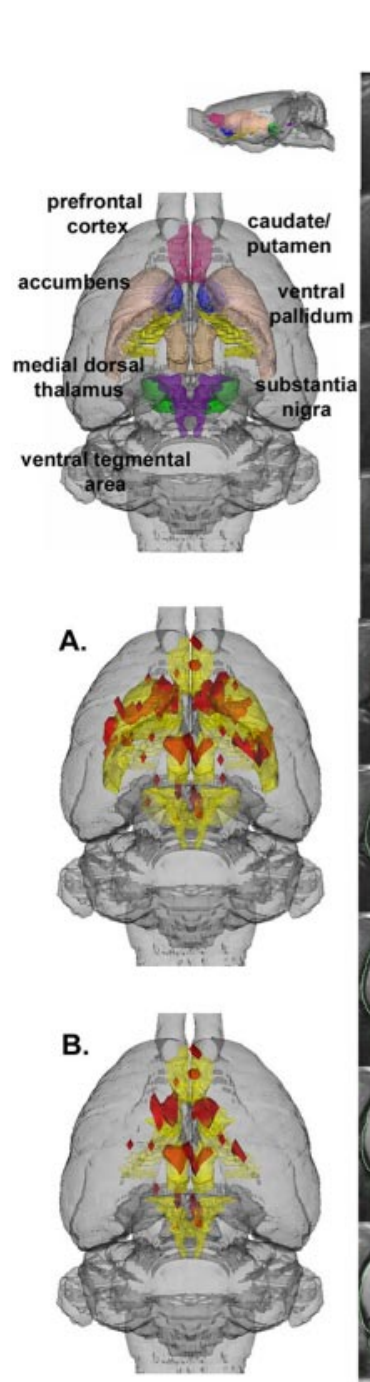

cumbens, ventral tegmental area, and medial dorsal thalamus for lactating dams given cocaine compared with pups-dams and cocaine-virgin conditions. Although there were significantly fewer positive BOLD voxels in the prefrontal cortex and caudate-putamen in the cocaine-dam condition as shown in Figure 4, those volumes that were activated had a similar BOLD signal change compared with pups-dam and virgin-female conditions. There were no significant changes in negative BOLD signal intensity in virgin females given cocaine for either the accumbens or prefrontal cortex.

\section{Discussion}

The present data show that pup suckling is

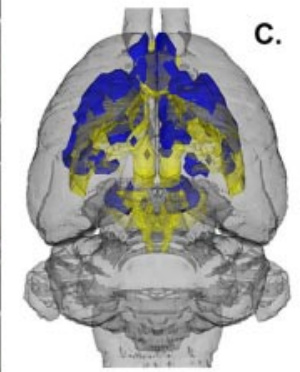
a robust stimulus for activating the mesocorticolimbic system. This dopaminergic pathway from the ventral tegmental area to the accumbens and prefrontal cortex system is involved in reward seeking and may help to strengthen the pup-dam bond. The simultaneous activation of the nigrostriatal and mesocorticolimbic dopamine systems was anticipated with cocaine administration, because this drug blocks reuptake and enhances dopaminergic ac-

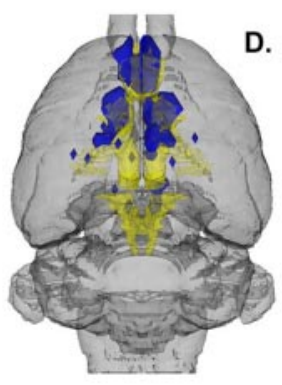

D. tivity everywhere. However, the coordinated activation of these parallel dopaminergic systems with pup stimulation was unexpected and may reflect the natural pattern of brain activation with reinforcing stimuli. These dorsal and ventral striatal pathways comprise the basal ganglia that, through thalamic and cortical connections, facilitate or inhibit expression of motor, cognitive, and behavioral processes. The reciprocal interactions between these striatal pathways may be coordinated through dopamine (Groenewegen et al., 2003).

The mesolimbic dopamine system appears to be a critical neurochemical pathway in the anticipatory and consummatory aspects of maternal behavior. Dams substantia nigra; PFC, prefrontal cortex; $A C b$, accumbens; VP, ventral pallidum; MD, medial dorsal thalamus.

uncertainty of 0.05 . Additionally, the individual subjects used a false-positive detection filter set at an overall uncertainty of 0.05 . The pups-dam condition induced significantly greater voxel activation for all VOIs shown than the cocaine-dam condition. Conversely, the cocaine-dam condition induced a significantly greater number of negative BOLD voxels compared with both pups-dam and cocaine-virgin in all VOIs shown than the pupsdam condition.

The change in BOLD signal intensity over time for each of the VOIs reported in Figure 4 is shown in Figure 5. Within 6-12 sec of stimulus presentation for all conditions, there was an increase and/or decrease in BOLD signal that persisted for the duration of the $5 \mathrm{~min}$ test period. There were no ostensible differences in the time course of positive BOLD signal between pups-dam and cocaine-virgin conditions. In both stimulus conditions, the positive BOLD signal changes ranged between $2-4 \%$. In contrast, the time course of positive BOLD signal $(\geq 1 \%)$ was less in the ac- between P4 and P10 show elevated extracellular dopamine levels in the nucleus accumbens in response to pup suckling (Hansen et al., 1993). Most recently, Champagne et al. (2004) showed increased extracellular dopamine in the shell of the accumbens of lactating dams in anticipation of pup licking and grooming. Lesioning the VTA-accumbens circuit with dopamine-depleting 6-hydroxydopamine reduces pup retrieval (Hansen et al., 1991a,b). Lactating dams treated with different dopamine receptor antagonists in the nucleus accumbens show a reduction in several measures of maternal behavior (Giordano et al., 1990; Stern and Taylor, 1991; Keer and Stern, 1999). Collectively, these findings support the notion that pup activation of the dopamine system is an important reinforcer in the anticipatory aspect of dams seeking pups and in the consummatory aspect of maternal behavior such as pup retrieval and pup licking and grooming. In a recent study using fMRI, Lorberbaum et al. (2002) reported 

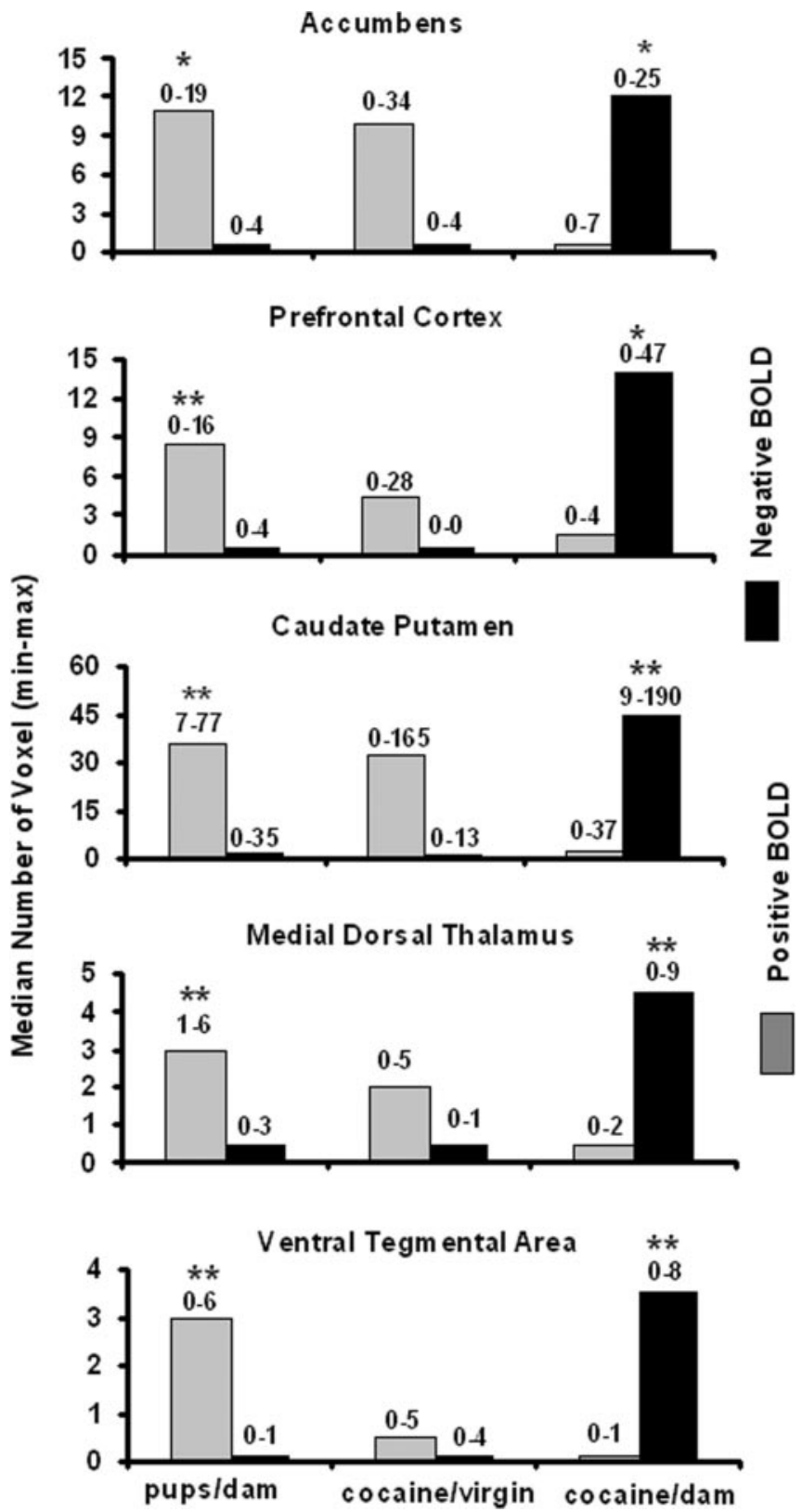

Figure 4. Volumes of activation. The bar graphs show the median number of voxels with a significant change in BOLD signal intensity with a $p$ value $<0.05$ after applying the falsepositive filter for the volumes of interest noted. Numbers above each bar represent the minimum and maximum from six animals. There was no significant differences in the number of positive (gray) or negative (black) voxels between pups-dam and cocaine-virgin conditions. ${ }^{*} p<0.05$; ${ }^{* *} p<0.01$, using a Bonferroni correction for a three-group comparison.

activation of the mesocorticolimbic system in mothers in response to their infants' recorded cries. This finding suggests that activation of the mothers' reward system may be generalized to a variety of infant-specific sensory stimuli and not just the tactile sensation of suckling.

Pup suckling stimulates the same mesocorticolimbic dopaminergic systems as cocaine in virgin females, evidence that both reinforcing stimuli elicit similar brain mechanisms regarding motivation and reward. However, the reproductive condition of the female appears to be a critical determinant in predicting the effect of cocaine on brain activation. Virgin females of the same age as the maternal, lactating females show a robust positive BOLD signal change in the reward system in response to cocaine.

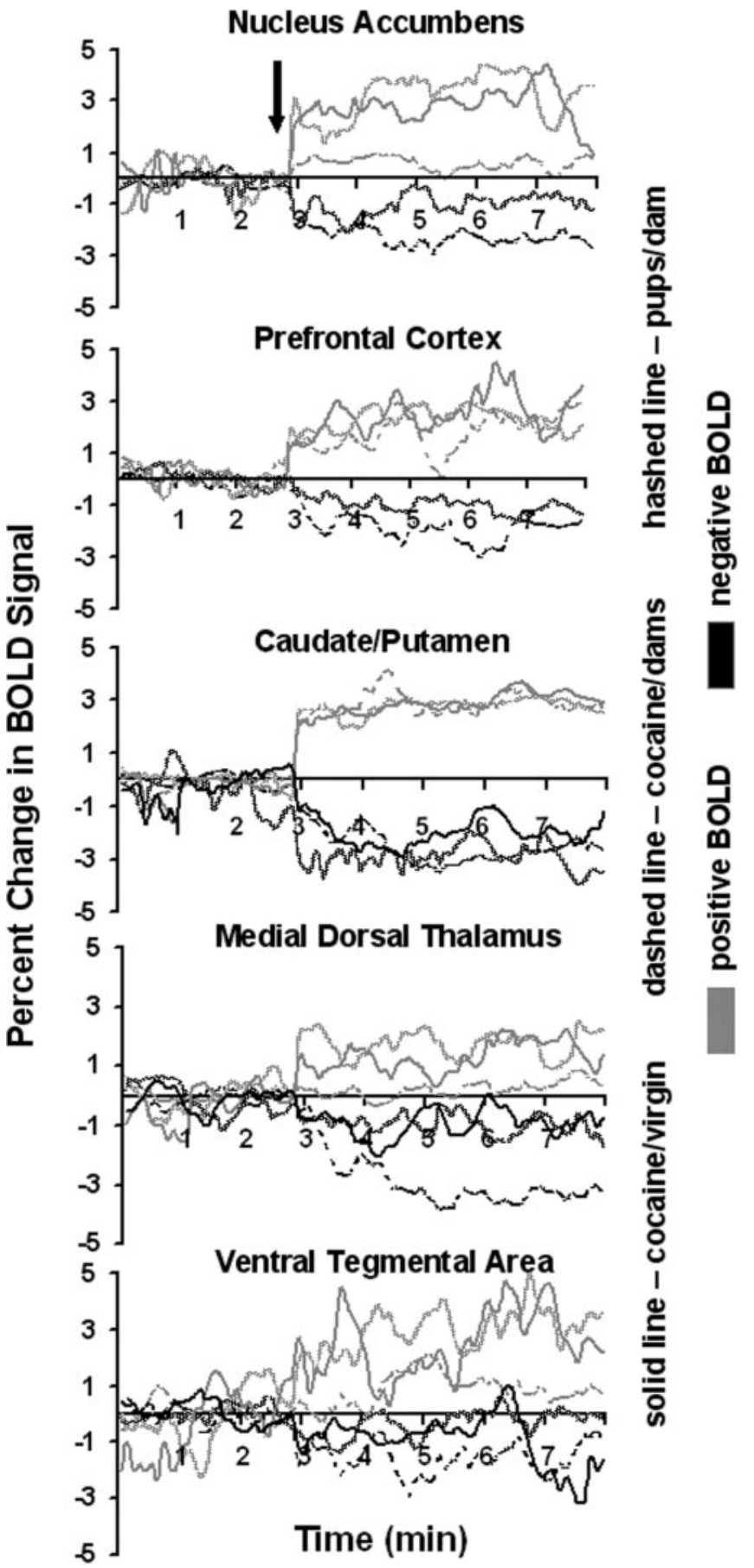

Figure 5. BOLD signal changes over time. The time course plots show the percentage change in positive (gray) and negative (black) BOLD signal after stimulus presentation (arrow) for the corresponding volumes of interest. The stimuli were either exposure to pups for the duration of $5 \mathrm{~min}$ or a bolus ICV injection of cocaine $(20 \mu \mathrm{g} / 20 \mu \mathrm{l})$ followed by 5 min of data acquisition.

However, the same dose of cocaine given to lactating dams causes a predominately negative BOLD response in the reward pathway, reflecting a suppression of synaptic and neuronal activity. The difference is probably attributable to neuroadaptive changes over the reproductive period of birth and early lactation that fosters mother-infant bonding, in part by making suckling a rewarding experience. Virgin females trained to self-administer cocaine and subsequently bred show a reduction in self-administration during early lactation (Hecht et al., 1999). As noted previously, the reinforcing properties of pups, as measured with conditioned 
place preference, exceeds that of cocaine in early lactation (Mattson et al., 2001, 2003).

The pronounced negative BOLD over most of the mesencephalic dopaminergic system in lactating dams exposed to cocaine is noteworthy. Functional MRI is a technique sensitive to the oxygenation status of hemoglobin and therefore produces images that reflect changes in cerebral blood flow and volume. With enhanced synaptic and neuronal activity, there is a compensatory increase in blood flow and oxygenated hemoglobin causing a positive BOLD change in magnetic resonance signal (Ogawa et al., 1993; Logothetis et al., 2001). Whereas positive BOLD signal reflects increased brain activity, the appearance of negative BOLD is less well understood. There are three primary reasons for the occurrence of negative BOLD: (1) the rate of oxygen consumption $\left(\mathrm{CMRO}_{2}\right)$ from areas of high synaptic and neuronal activity exceeds compensatory blood flow; (2) "vascular steal" (i.e., preferential flow to the most metabolically active areas leaving the abandoned areas facing less blood flow in the presence of a constant or slightly elevated $\mathrm{CMRO}_{2}$ ) (Harel et al., 2002); and (3) a reduction in brain activity and blood flow from basal levels (Shmuel et al., 2002). The robust negative BOLD signal observed in these studies when lactating dams are exposed to cocaine is interpreted as brain deactivation. To use the phrase "inhibition of brain activity" would be incorrect, because synaptic inhibition in the form of inhibitory postsynaptic potentials is an active process accompanied by enhanced oxidative metabolism. Brain deactivation suggests reduced synaptic and neuronal activity without drawing attention to a biophysical mechanism. It is unlikely that the cocaine-induced negative BOLD signal in the mesencephalic dopamine system is attributable to excessive brain activation with deficient compensatory blood flow, because it was not seen in the virgin females. This same argument applies to the possible mechanism of vascular steal(i.e., why would you see it for a whole neural pathway in one reproductive condition and not another?).

This raises the critical question of why cocaine stimulates the reward system and is an effective reinforcer in virgin rats but is less effective, even inhibitory, in dams during early lactation. If pups and cocaine activate a common neural substrate as the present findings would indicate, then the response of one would affect the other depending on the salience and magnitude of the reinforcing stimuli (Mattson et al., 2001). Introduction of a highly addictive drug such as cocaine into a critical reproductive period of social bonding might be expected to usurp or disrupt the natural stimulus-reward relationship between pups and dams (Panksepp et al., 2002). Instead, evolution may have provided dams with a resistance against competing hedonic stimuli during early lactation, assuring the salience of pup seeking over other rewards (Mattson et al., 2001). Oxytocin, a neuropeptide released during parturition and lactating, may be one factor contributing to this preference for pups over cocaine (Insel, 2003). There is considerable evidence showing that oxytocin is necessary for the onset of maternal behavior, social memory, pair bonding, and affiliative behaviors. Because pup suckling as a rewarding social stimulus is linked to the mesolimbic dopaminergic system, it could be hypothesized that oxytocin promotes affiliation by enhancing dopamine neurotransmission. Indirect evidence in support of this notion comes from studies reporting that oxytocin interferes with the mesolimbic dopamine system by blocking or suppressing many of the behavioral effects of cocaine (Kovács et al., 1998). For example, pretreating rats with oxytocin reduces hyperactivity and stereotypy triggered by acute injections of cocaine (Kovács et al., 1990). Therefore, the enhanced release of oxytocin during nursing may function to promote bond forma- tion between mother and pup while reducing the sensitivity of the mesolimbic dopamine system to the stimulatory and rewarding effects of cocaine.

\section{References}

Brevard ME, Duong TQ, King JA, Ferris CF (2003) Changes in MR signal intensity during hypercapnic challenge under conscious and anesthetized conditions. Magn Reson Imaging 21:995-1001.

Champagne FA, Chretien P, Stevenson CW, Zhang TY, Gratton A, Meaney MJ (2004) Variations in nucleus accumbens dopamine associated with individual differences in maternal behavior in the rat. J Neurosci 24:4113-4123.

Febo M, Segarra A, Tenney J, Sullivan R, Brevard M, Duong TQ, Ferris CF (2004) Imaging cocaine-induced changes in the reward system in conscious rats. J Neurosci Methods 139:167-176.

Fleming AS, Korsmit M, Deller M (1994) Rat pups are potent reinforcers to the maternal animal: effects of experience, parity, hormones and dopamine function. Psychobiology (Austin, Tex) 22:44-53.

Genovese CR, Lazar NA, Nichols T (2002) Thresholding of statistical maps in functional neuroimaging using the false discovery rate. NeuroImage 15:870-878.

Giordano B, Johnson AE, Rosenblatt JS (1990) Haloperidol-induced disruption of retrieval behavior and reversal with apomorphine in lactating rats. Physiol Behav 48:211-214.

Groenewegen HJ, van den Heuvel OA, Cath DC, Voorn P, Veltman DJ (2003) Does an imbalance between the dorsal and ventral striatopallidal systems play a role in Tourette's syndrome? A neuronal circuit approach. Brain Dev 25 [Suppl 1]:S3-S14.

Hansen S, Harthon C, Wallin E, Löfberg L, Svensson K (1991a) The effects of 6-OHDA-induced dopamine depletions in the ventral or dorsal striatum on maternal and sexual behavior in the female rat. Pharmacol Biochem Behav 39:71-77.

Hansen S, Harthon C, Wallin E, Löfberg L, Svensson K (1991b) Mesotelencephalic dopamine system and reproductive behavior in the female rat: effects of ventral tegmental 6-hydroxydopamine lesions on maternal and sexual responsiveness. Behav Neurosci 105:588-598.

Hansen S, Bergvall ÅH, Nyiredi S (1993) Interaction with pups enhances dopamine transmission in the ventral striatum of maternal rats: a microdialysis study. Pharmacol Biochem Behav 45:673-676.

Harel N, Lee SP, Nagaoka T, Kim DS, Kim SG (2002) Origin of negative blood oxygen level-dependent fMRI signals. J Cereb Blood Flow Metab 22:908-917.

Hecht GS, Spear NE, Spear LP (1999) Changes in progressive ratio responding for intravenous cocaine throughout the reproductive process in female rats. Dev Psychobiol 35:136-145.

Huang W, Sullivan Jr JM, Ludwig R, Kulkarni P, Zhang JQ, King JA (2004) Stair-stepped removal via automatic linearization for marching cubes formulations. Paper presented at 12th Annual Conference of the International Society of Magnetic Resonance in Medicine, Kyoto, Japan.

Insel TR (2003) Is social attachment and addictive disorder? Physiol Behav 79:351-357.

Keer SE, Stern JM (1999) Dopamine receptor blockade in the nucleus accumbens inhibits maternal retrieval and licking, but enhances nursing behavior in lactating rats. Physiol Behav 67:659-669.

Kovács GL, Sarnyai Z, Babarczy E, Szabó G, Telegdy G (1990) The role of oxytocin-dopamine interactions in cocaine-induced locomotor hyperactivity. Neuropharmacology 29:365-368.

Kovács GL, Sarnyai Z, Szabó G (1998) Oxytocin and addiction: a review. Psychoneuroendocrinology 23:945-962.

Lee A, Li M, Watchus J, Fleming AS (1999) Neuroanatomical basis of maternal memory in postpartum rats: selective role for the nucleus accumbens. Behav Neurosci 113:523-538.

Lee A, Clancy S, Fleming AS (2000) Mother rats bar-press for pups: effects of lesions of the MPOA and limbic sites on maternal behavior and operant responding for pup-reinforcement. Behav Brain Res 108:215-231.

Logothetis NK, Pauls J, Augath M, Trinath T, Oeltermann A (2001) Neurophysiological investigation of the basis of the fMRI signal. Nature 412:150-157.

Lorberbaum JP, Newman JD, Horwitz AR, Dubno JR, Lydiard RB, Hamner MB, Bohning DE, George MS (2002) A potential role for thalamocingulate circuitry in human maternal behavior. Biol Psychiatry 51:431-445. 
Ludwig R, Bogdanov G, King J, Allard A, Ferris CF (2003) A dual RF resonator for high-field functional magnetic resonance imaging of small animals. J Neurosci Methods 132:125-135.

Magnusson JE, Fleming AS (1995) Rat pups are reinforcing to the maternal rat: role of sensory cues. Psychobiology (Austin, Tex) 231:69-75.

Mattson BJ, Williams SE, Rosenblatt JS, Morrell JI (2001) Comparison of two positive reinforcing stimuli: pups and cocaine throughout the postpartum period. Behav Neurosci 115:683-694.

Mattson BJ, Williams SE, Rosenblatt JS, Morrell JI (2003) Preferences for cocaine- or pup-associated chambers differentiates otherwise behaviorally identical postpartum maternal rats. Psychopharmacology (Berl) 167:1-8.

Numan M (1994) Maternal behavior. In: The physiology of reproduction, Ed 2 (Knobil E, Neill JD, eds), pp 221-302. New York: Raven.

Ogawa S, Menon RS, Tank DW, Kim SG, Merkle H, Ellermann JM, Ugurbil K (1993) Functional brain mapping by blood oxygenation level-dependent contrast magnetic resonance imaging. Biophys J 64:803-812.

Panksepp J, Knutson B, Burgdorf J (2002) The role of brain emotional systems in addictions: a neuro-evolutionary perspective and new "selfreport" animal model. Addiction 97:459-469.

Paxinos G, Watson C (1991) The rat atlas in stereotaxic coordinates, Ed 2. New York: Academic

Pedersen CA, Caldwell JD, Walker C, Ayers G, Mason GA (1994) Oxytocin activates the postpartum onset of rat maternal behaviour in the ventral tegmental and medial preoptic areas. Behav Neurosci 108:1163-1171.

Petruccelli JD, Nandram B, Chen M (1999) Applied statistics for engineers and scientists, pp 535-536. Englewood Cliffs, NJ: Prentice Hall.

Shmuel A, Yacoub E, Pfeuffer J, Van de Moortele PF, Adriany G, Hu X, Ugurbil K (2002) Sustained negative BOLD, blood flow and oxygen consumption response and its coupling to the positive response in the human brain. Neuron 36:1195-1210.

Stern JM, Taylor LA (1991) Haloperidol inhibits maternal retrieval and licking, but facilitates nursing behavior and milk ejection in lactating rats. J Neuroendocrinol 3:591-596.

Swanson LW (1998) Brain maps: structure of the rat brain, Ed 2. Amsterdam: Elsevier.

Tenney JR, Duong TQ, King JA, Ludwig R, Ferris CF (2003) Corticothalamic modulation during absence seizures: a functional MRI approach. Epilepsia 44:1133-1140.

Wu Z, Sullivan Jr JM (2003) Multiple material marching cubes algorithm. Int J Numer Methods Engin 58:189-207.

Zhang JQ, Sullivan Jr JM, Kulkarni PP, Brevard M, Benz U, Yu HL (2003) Intermodality registration via interactive graphics coupled with A.I.R. intramodality registration. Paper presented at 11th Annual Conference of the International Society of Magnetic Resonance in Medicine, Toronto, Ontario, Canada. 